\title{
Stochastic Process on CL Multiwavelet
}

\author{
Jaka Sembiring Kageo Akizuki \\ Dept. of Electrical Engineering; Waseda University, \\ 3-4-1, Ohkubo, Shinjuku-ku, Tokyo 169-8555, Japan
}

\begin{abstract}
This paper describes an early attempt to build a new multi-scale stochastic model on multiwavelet. Previously such stochastic model are defined either on a single or multiple binary tree as well as on an ordinary wavelet structure. Several interesting results are obtained due to the specific properties of multiwavelet which are different from its wavelet counterpart. Smoothing of a certain fractal signal on the proposed model will be given.
\end{abstract}

\section{Introduction}

Recently research topics focusing on the descriptions and applications of the multi-scale stochastic process has gained momentum thanks to the nice properties of such model on dealing with fractal signal $[1,2,3,4$, $5,6]$. Earlier model is defined on a single binary tree, see e.g. [1, 2], then [4] moves further with model on wavelet. The authors gave another model from different point of view in [6]. On the other development, the wavelet theory gets another impetus driven from the introduction of what the so called multiwavelet that can be seen e.g. in $[7,8]$. This multiwavelet has been known to have several advantages over ordinary wavelet such as short support, symmetry and orthogonality. In this paper those advantages will be further investigated in relation to the stochastic modeling.

The basic construction of a multi-scale stochastic model is a state space evolving on a dyadic tree $\mathcal{T}$, given in Fig. 1. Upon going down towards the finest scale $N$, the resolution doubles from scale to scale, and vice versa. In wavelet theory it has been known that the Haar wavelet decomposition or a pure decimator behave just like the upward evolution, and the synthesis process is equivalent to the downward path. This paper expands the model further from ordinary wavelet to multiwavelet structure.

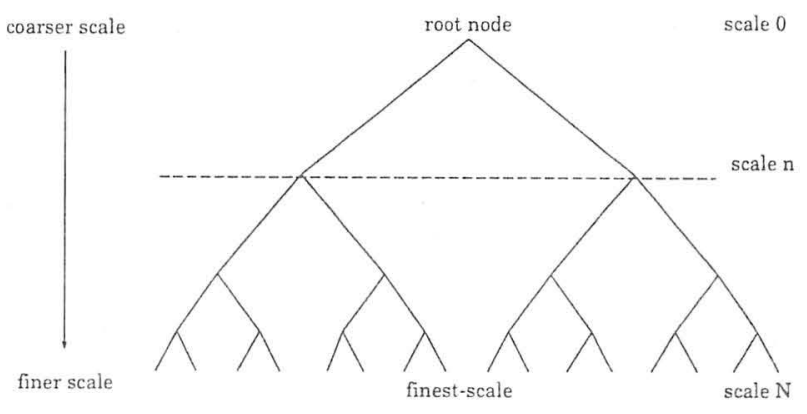

Figure 1: Dyadic tree $\mathcal{T}$

\section{Multiwavelet}

\subsection{Multi-resolution Analysis}

Multiwavelet theory as an addition to the body of wavelet theory, falls on the realm of of multi-resolution analysis (MRA). But instead of assuming single scaling function $\phi(t)$, the multiwavelet, extends the MRA by $N$ scaling function $\Phi(t)=\left[\phi_{1}(t), \cdots, \phi_{N}(t)\right] \in \mathbb{L}^{2}(\mathbb{R})^{N}$, such that subspaces

$$
V_{j} \equiv \overline{\operatorname{span}\left\{2^{j / 2} \phi_{i}\left(\frac{t}{2 j}-k\right): 1 \leq i \leq N, k \in \mathbb{Z}\right\}}
$$

are nested, i.e

$$
\cdots \subset V_{2} \subset V_{1} \subset V_{0} \subset V_{-1} \subset V_{-2} \subset \cdots
$$

and the conditions

$$
\overline{\bigcap_{j \in \mathbb{Z}} V_{j}}=\mathbb{L}^{2}(\mathbb{R}) \text { and } \bigcup_{j \in \mathbb{Z}} V_{j}=\{0\}
$$

hold. It is understood that all scaling functions $\phi_{1}(t), \cdots, \phi_{N}(t)$ lies in $V_{0}$ where $V_{0} \subset V_{1}$ whose basis are $\left\{\sqrt{2} \phi_{i}(2 t-k): 1 \leq i \leq N, k \in \mathbb{Z}\right\}$ that the following two-scale matrix dilation equation is satisfied

$$
\Phi(t)=\sqrt{2} \sum_{k} G_{k} \Phi(2 t-k)
$$

where $G_{k \in \mathbb{Z}} \in l^{2}(\mathbb{Z})^{N \times N}$ are $N \times N$ scaling coefficients. For orthonormal scaling function, it can be shown that

$$
\sum_{k} G_{k} G_{2 l+k}^{T}=I_{N} \delta_{0, l}, \text { for } l \in \mathbb{Z}
$$


Let $W_{j}$ be the complement spaces of $V_{j}$ in $V_{j-1}$ such that

$$
V_{j-1}=V_{j} \oplus W_{j} \text { and } V_{j} \bigcap W_{j}=0
$$

Let $\Psi(t)=\left[\psi_{1}(t), \cdots, \psi_{N}(t)\right] \in \mathbb{L}^{2}(\mathbb{R})^{N}$, then define

$$
W_{j} \equiv \overline{\operatorname{span}\left\{2^{j / 2} \psi_{i}\left(\frac{t}{2 j}-k\right): 1 \leq i \leq N, k \in \mathbb{Z}\right\}}
$$

$\psi(t)$ is called a multiwavelet if $\left\{\psi_{i}(t-k): 1 \leq i \leq\right.$ $N, k \in \mathbb{Z}\}$ is Riesz basis of $W_{0}$. The multiscaling and multiwavelet function satisfy two-scale matrix wavelet equation

$$
\Psi(t)=\sqrt{2} \sum_{k} H_{k} \Phi(2 t-k)
$$

where $H_{k \in \mathbb{Z}} \in l^{2}(\mathbb{Z})^{N \times N}$ are $N \times N$ wavelet coefficients. In orthogonal multi-resolution analysis the $W_{j}$ are orthogonal complement to $V_{j}$ in $V_{j-1}$. This fact leads to the following relation

$$
\sum_{k} G_{k} H_{2 l+k}^{T}=0_{N}, \text { for } l \in \mathbb{Z}
$$

In particular, the matrix $H_{k}$ in orthonormal wavelet system satisfies the condition below

$$
\sum_{k} H_{k} H_{2 l+k}^{T}=I_{N} \delta_{0, l}, \text { for } l \in \mathbb{Z}
$$

The pair of Eqs. (4) and (8) can be realized with matrix filter banks operating on $N$ input data stream and producing $2 N$ decimated by 2 output streams. If $x(t) \in V_{0}$ denotes input signal then multiwavelet decomposition of this signal can be obtained by recursively calculate the following

$$
\begin{aligned}
v_{j, k} & =\sum_{m} G_{m-2 k} v_{j-1, m} \\
w_{j, k} & =\sum_{m} H_{m-2 k} v_{j-1, m}
\end{aligned}
$$

The original signal $x(t)$ can be found through synthesis equation

$$
v_{j-1, k}=\sum_{m} \tilde{G}_{k-2 m}^{T} v_{j, m}+\sum_{m} \tilde{H}_{k-2 m}^{T} w_{j, m}
$$

If $\phi_{1}(t-k), \cdots, \phi_{N}(t-k), k \in \mathbb{Z}$ form an orthogonal basis of $V_{0}$ then the multiwavelet system will be orthogonal.

This paper will focus on a certain type of orthogonal multiwavelet known as Chui-Lian (CL) pair multiwarelet whose coefficients $G$ and $H$ are determined as

$$
\begin{aligned}
& G_{0}=\frac{1}{2 \sqrt{2}}\left[\begin{array}{cc}
1 & -1 \\
\frac{\sqrt{7}}{2} & -\frac{\sqrt{7}}{2}
\end{array}\right] \quad G_{1}=\frac{1}{2 \sqrt{2}}\left[\begin{array}{ll}
2 & 0 \\
0 & 1
\end{array}\right] \\
& G_{2}=\frac{1}{2 \sqrt{2}}\left[\begin{array}{cc}
1 & 1 \\
-\frac{\sqrt{7}}{2} & -\frac{\sqrt{7}}{2}
\end{array}\right] \\
& H_{0}=\frac{1}{4 \sqrt{2}}\left[\begin{array}{cc}
2 & -2 \\
-1 & 1
\end{array}\right] \quad H_{1}=\frac{1}{4 \sqrt{2}}\left[\begin{array}{cc}
-4 & 0 \\
0 & 2 \sqrt{7}
\end{array}\right] \\
& H_{2}=\frac{1}{4 \sqrt{2}}\left[\begin{array}{ll}
2 & 2 \\
1 & 1
\end{array}\right]
\end{aligned}
$$

This choice is motivated by orthogonality of the prefiltering needed in the process.

\subsection{Pre-filtering}

As described in the previous subsection, the multiwavelet system needs $N$ row of input data stream, and in particular choosing CL wavelet results in $N=2$ input data streams. The choice of such multiwavelet is related to the properties of the pre-filtering involved in multiwavelet system. For simplicity this paper will use critical sampling method described in [7] as a prefiltering method. This is due to the fact that the covariance matrix of pre-filtering result retain diagonal matrix form needed to maintain orthogonality in the subsequent derivation.

The pre-filtering procedure involves a matrix $P_{0}=$ $\left[\begin{array}{cc}1 / 4 & 1 / 4 \\ -1 / 6 & 1 / 6\end{array}\right]$ to obtained data stream at. level 0 written as

$$
v_{0, k}=P_{0}\left[\begin{array}{c}
v_{2 k} \\
v_{2 k+1}
\end{array}\right]
$$

so that high pass filtering $\sum_{m} H_{m-2 k} v_{0, m}$ is zero. For input comprises a signal corrupted by zero-mean noise $X_{k}=D_{k}+v_{k}$, it can be obtained that the covariance matrix cov $\left[X_{k} X_{k}\right]$ at level 0 for $\left[v_{0,0}^{0}, v_{0,0}^{1}, v_{0,1}^{0}, v_{0,1}^{1}\right]$ remain diagonal

$$
\operatorname{cov}\left[X_{k} X_{k}\right]=\sigma_{\epsilon}^{2}\left[\begin{array}{cccc}
0.125 & 0 & 0 & 0 \\
0 & 0.15 & 0 & 0 \\
0 & 0 & 0.125 & 0 \\
0 & 0 & 0 & 0.15
\end{array}\right]
$$

where $\sigma_{\epsilon}^{2}$ is the variance of the noise. With this property and the orthogonality of the multiwavelet, one can retain the orthogonality throughout the system.

\section{Stochastic Model}

Stochastic model based on wavelet decomposition has been covered in [4]. The extension to multiwavelet structure, given apparent advantages of multiwavelet, is the main topics in this paper. As described in the previous section, the multiwavelet transformation consists of filtering $G_{k \in \mathbb{Z}} \in l^{2}(\mathbb{Z})^{N \times N}$ and $H_{k \in \mathbb{Z}} \in l^{2}(\mathbb{Z})^{N \times N}$ 
followed by a decimation by two. In this paper, the process filtering $G_{k}$ with such decimation will be referred as $a$, and $\beta$ for $H_{k}$. filter respectively. In other words the operators $\alpha$ and $\beta$ works from $l^{2}\left(\mathbb{Z}\right.$ to $l^{2}(2 \mathbb{Z})$. For given signal or vector $X_{n}$ at level $n$ then $a X_{n}$ is the coarse approximation at level $n+1$, whereas $\beta X$ yields the detail required upon going from one level to the next. From Eq. (12), the reverse process, i.e. going from coarse to fine involves adjoint operators $\bar{\alpha}$ and $\bar{\beta}$ for filter $\tilde{G}_{k}$ and $\tilde{H}_{k}$ respectively. From Eqs. (5), (9), (10), and (12), it is clear that the following conditions are satisfied

$$
\begin{aligned}
& \bar{\alpha} \alpha+\bar{\beta} \beta=1 \\
& \bar{\alpha} \alpha=I=\bar{\beta} \beta \\
& \alpha \bar{\beta}=0=\beta \bar{\alpha}
\end{aligned}
$$

Now the relation of the transformation above with the stochastic modeling can be explained as follows. Let us define a multi-scale stochastic model written in the following forms

$$
\begin{aligned}
X_{n+1} & =\left(\bar{\alpha}_{n} A_{n}+\bar{\beta}_{n} B_{n}\right) X_{n}+W_{n+1} \\
Y_{n+1} & =C X_{n}+V_{n}
\end{aligned}
$$

where $n$ denotes scale index increasing towards finest scale $N, \bar{\alpha}, \bar{\beta}$ are multiwavelet transformation adjoint operators. $X_{n}$ is the state vector with stationary white noise $W_{n}$, and $Y_{n}$ is the observation vector also with stationary white noise $V_{n}$. These noises are independent each other. The variables $A_{n}, B_{n}$ and $C_{n}$ are matrices with appropriate dimensions. It can be seen that the model written in Eq. (17) can be associated with a synthesis process of multiwavelet transformation, see Eq. (12), such that the evolution of Eq. (17) is towards finer scale through adjoint operators $\bar{\alpha}$ and $\bar{\beta}$. The initial process at $X_{0}$ is called the root node, and the finest level at $X_{N}$ is referred to leaf nodes.

Once transformation or decomposition through multiwavelet is carried out, the coefficients becomes the data set. Then there exist several facts as follows

(1) Let $w_{n}$ consists of $\mathcal{W}_{n}=\{\alpha, \beta\}$ for $0 \leq n<N$.

(2) Then $w_{n} X_{n}$ is linked to the coarser scale through $w_{n-1} X_{n-1}$, and it is conditionally independent given $X_{n-1}$.

(3) $w_{n} Y_{n}$ is linked to $X_{n}$ through $w_{n} X_{n}$, and it is conditionally independent given $X_{n}$.

Following the term used in [4], one can get a smoother on each board of coefficient decomposition where the smoothing process in each board is independent. Different from wavelet structure where there are $N$ board depending on the filter taps, in multiwavelet decomposition there are at least twice board as much. This can be seen in Eq. (1) where $\Phi(t)=$ $\left[\phi_{1}(t), \cdots, \phi_{N}(t)\right] \in \mathbb{L}^{2}(\mathbb{R})^{N}, N=2$ for CL multiwavelet.

With Chui-Lian (CL) multiwavelet, the two rows of input signal will have diagonal matrix correlation. This fact in addition to the orthogonality of the multiwavelet transformation will lead us to the implementation of a smoother on model Eq. (17) by taking advantages of the fast multi-scale smoothing algorithm [1]. It is shown through simulation that in case of smoothing of a typical fractal signal, significant improvement over previously announced model can be achieved.

\section{Simulation}

The subject of this section is the smoothing procedure of a certain fractal signal using the method described in the previous section. Assume that there exists an observation signal corrupted by white Gaussian noise $(\mathrm{SNR}=\sqrt{2})$ depicted as dashed line in Figs. 2, 3, and 4. The true signal of this noisy observation is generated through multi-scale system elaborated in $[1,2]$ and given by the dotted line in those figures. Then the smoothing results on a variety of wavelets are given by solid line. Upon examining the graphs closely, a smoothing based on Haar wavelet reveals some blocky nature mentioned in [1]. A better result can be achieved through Daubechies4 wavelet, see Fig. 3, refer also [4]. Fig. 4 depicts the smoothing of the signal on the CL multiwavelet structure which shows further improvement over the two previously announced methods.

The quality of the proposed smoothing result based on CL multiwavelet are given numerically in Table 1 , as well as the result using Haar and Daubechies4 wavelet for comparison. The $M S E$ value of each process shows that the proposed method gives better performance. Other important quality measurement is the Bhattacharrya distance which is given to show the closeness of two random vector. It is based on the following formula

$$
B_{d}\left(R_{1}, R_{2}\right)=\frac{1}{2} \ln \left[\left|\frac{1}{2}\left(R_{1}+R_{2}\right)\right|\right]-\frac{1}{4} \ln \left[\left|\left(R_{1} R_{2}\right)\right|\right]
$$

where $R_{1}, R_{2}$ denote the covariance matrices of both random vectors, and $|R|$ is the determinant of the ma$\operatorname{trix} R$. Now given two random vectors with probability of obtaining a sample from either vectors is $1 / 2$, then the probability of error of deciding from which process the sample was is bounded by the following

$$
P \text { (error) } \leq \frac{1}{2} \exp ^{-B_{d}\left(R_{1}, R_{2}\right)}
$$

Bhattacharrya distance and probability of error also confirm that the smoothing based on the CL multiwavelet outperforms ordinary wavelet base. 


\begin{tabular}{|c|c|c|c|}
\hline Wavelet & MSE & $B_{d}$ & $P$ (error) \\
\hline \hline Haar & 1.035391 & 6.695519 & 0.000618 \\
Daubechies4 & 1.032211 & 3.371325 & 0.017172 \\
Chui-Lian & 1.026031 & 1.849849 & 0.078630 \\
\hline
\end{tabular}

Table 1: Quality of smoothing

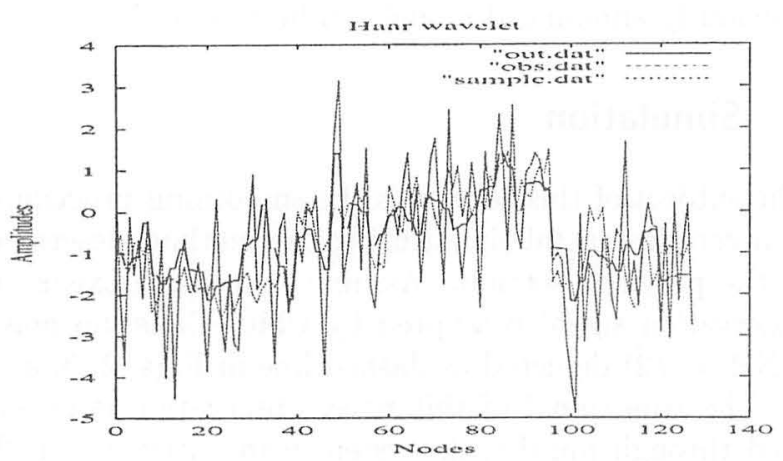

Figure 2: Smoothing with Haar wavelet

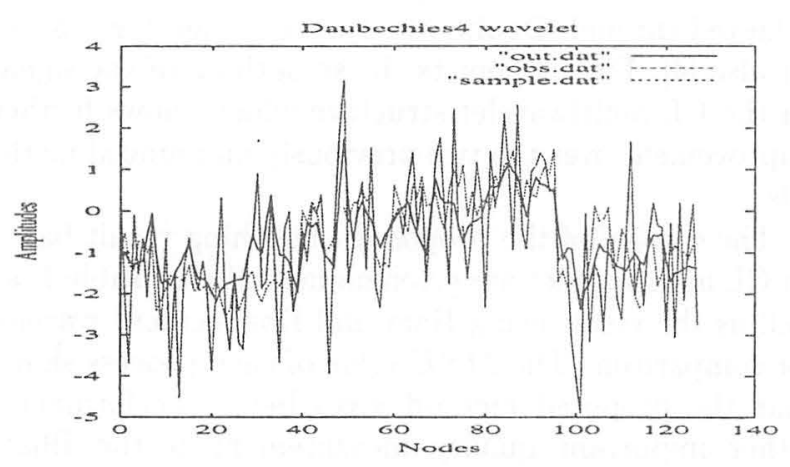

Figure 3: Smoothing with Daubechies4 wavelet

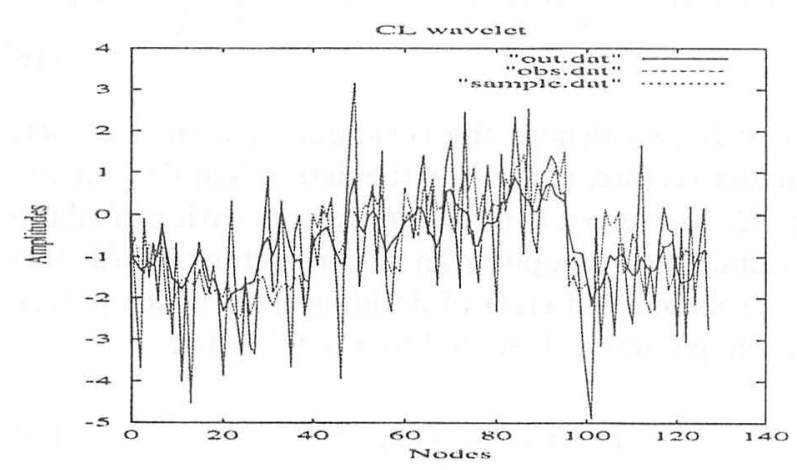

Figure 4: Smoothing with Chui-Lian multiwavelet

\section{Conclusion}

This paper described an early attempts on modeling of stochastic system evolving on the multiwavelet structure. Simulation results hinted that several improvements over previously published results can be achieved through the proposed method.

\section{References}

[1] K. C. Chou, A. S. Willsky, and A. Benveniste, "Multiscale recursive estimation, data fusion, and regularization," IEEE Trans. on Automatic Control, vol. 39, no. 3, pp. 464-478, 1994.

[2] K. C. Chou, A. S. Willsky, and R. Nikoukhah, "Multiscale systems, Kalman filters, and Riccati equations," IEEE Trans. on Automatic Control, vol. 39, no. 3, pp. 479-492, 1994.

[3] M. Daniel and A. S. Willsky, "A multiresolution methodology for signal-level fusion and data assimilation with application in remote sensing," Proc. of IEEE, vol. 85, no. 1, pp. 164-180, 1997.

[4] E. D. Kolaczyk, Wavelets Methods for The Inversion of Certain Homogeneous Linear Operators in The Presence of Noisy Data. PhD thesis, Stanford University, October 1994.

[5] J. Sembiring and K. Akizuki, "Realization of multiscale stochastic process on multiple tree," Trans. of IEE of Japan, vol. 120-C, no. 2, pp. 194-200, 2000.

[6] J. Sembiring and K. Akizuki, "Application of multiple tree stochastic theory on estimating signal over network," in Proc. of IFAC SYSID 2000, (Santa Barbara, USA), June 2000.

[7] V. Strela et al., "The application of multiwavelet filter banks to signal and image processing," IEEE Trans. on IP, vol. 8, no. 2, pp. 548-563, 1999.

[8] V. Strela et al., "Application of multiwavelets to signal compression and denoising," in Proc. UK Symposium on Applications of Time-Frequency and Time-Scale Methods, 1995. 